\title{
Perfil dos cuidadores de pacientes com transtornos mentais do Hospital Universitário Walter Cantídio
}

\section{Profile of caregivers of patients with mental disorders of Hospital Universitário Walter Cantídio}

\author{
João Paulo Lima Santos ${ }^{1}$. Lis Caetano Nóbrega Costa Araújo ${ }^{2}$. Solangio Rodrigues Timbó ${ }^{2}$. Rodrigo Freitas \\ da Costa ${ }^{1}$. José Evangleyson de Paiva Girão ${ }^{3}$. Arthur Alcântara Lima ${ }^{4}$. Eugênio de Moura Campos ${ }^{5}$. \\ 1 Médico, Residente em Psiquiatria, Hospital Universitário Walter Cantídio (HUWC), Universidade Federal do Ceará (UFC), \\ Fortaleza, Ceará, Brasil. 2 Acadêmico(a) do curso de Medicina, Universidade Federal do Ceará (UFC), Fortaleza, Ceará, Brasil. \\ 3 Enfermeiro, Residente em Saúde Mental da Residência Multiprofissional em Saúde, Hospital Universitário Walter Cantídio \\ (HUWC), Universidade Federal do Ceará (UFC), Fortaleza, Ceará, Brasil. 4 Médico, Universidade Federal do Ceará (UFC), \\ Fortaleza, Ceará, Brasil. 5 Doutor em Farmacologia, Psiquiatra, Coordenador do programa de Psiquiatria do Hospital Universitário \\ Walter Cantídio (HUWC), professor adjunto de Psiquiatria pela Faculdade de Medicina, Universidade Federal do Ceará (UFC), \\ Fortaleza, Ceará, Brasil.
}

\section{RESUMO}

Objetivo: determinar o perfil dos cuidadores de pacientes com transtornos mentais acompanhados em ambulatórios de Psiquiatria Geral. Metodologia: estudo transversal realizado durante novembro e dezembro de 2016, sendo incluídos no estudo os cuidadores maiores de 18 anos que preenchiam a definição de cuidador informal. Eles responderam a um questionário sobre dados gerais e a sua função de cuidador. Os dados foram tabelados e analisados com o auxílio do Microsoft Excel ${ }^{\circledR}$ for Windows 2010 . Foram respeitados os princípios para os procedimentos éticos em pesquisas. Resultados: foram entrevistados 68 cuidadores. Notou-se predomínio do sexo feminino (80,9\%), ausência de orientações sobre a evolução dos transtornos apresentados (52,9\%) e falta de instruções sobre como auxiliar o paciente em casa (64,7\%). Os diagnósticos psiquiátricos mais comuns nos pacientes acompanhados foram depressão (33,8\%), transtornos psicóticos (30,9\%) e transtorno afetivo bipolar (10,3\%). As principais responsabilidades dos cuidadores envolviam comparecimento a consultas (96,6\%). Conclusão: percebe-se que ainda há carência de informações sobre transtornos mentais e sobre como manejar as dificuldades do cotidiano do paciente psiquiátrico. Com este perfil, podemos elaborar estratégias para fornecer mais atenção aos cuidadores e assim ajudá-los a lidar com os obstáculos associados aos transtornos mentais, diminuindo sua sobrecarga e proporcionando melhor cuidado ao paciente.

Palavras-chave: Cuidadores. Perfil de saúde. Saúde mental.

\section{ABSTRACT}

Objective: the goal of this research is to determine the profile of the caregivers currently helping patients with mental disorders followed up in General Psychiatry clinics. Methods: cross-sectional study conducted during November-December 2016. Caregivers older than 18 years who fulfilled the definition of informal caregiver were included in the study. They answered questions about general information and their responsibilities as caregivers. The data were tabulated and analyzed with Microsoft Excel ${ }^{\circledR}$ for Windows 2010. Ethical principles in research have been respected. Results: it was noted a predominance of female caregivers (80.9\%), lack of orientation about disorders (52.9\%) and lack of instructions on how to better care for patients at home (64.7\%). The most frequent psychiatric illnesses in the followed patients were depression (33.8\%), psychotic disorders (30.9\%) and bipolar disorder (10.3\%). The main caregiver responsibilities were accompanying patients to clinic visits $(96.6 \%)$ and administering their medication (82.4\%). Conclusion: considering the collected data, it is noticeable there is still need for education about mental disorders and how to manage the daily hardships of psychiatric patients. It's possible to plan strategies to provide more attention to caregivers and help them deal with the obstacles associated with mental illnesses, alleviating their overload and providing better care to patients.

Keywords: Caregivers. Health profile. Mental health.

Autor correspondente: João Paulo Lima Santos, Rua Juvenal de Carvalho, 710, Bairro de Fátima, Fortaleza, Ceará. CEP: 60050-220.Telefone: +55 85 99186-3689. E-mail: jplima.med@gmail.com

Conflito de interesses: Não há qualquer conflito de interesses por parte de qualquer um dos autores.

Recebido em: 24 Fev 2017; Revisado em: 23 Mar 2017; Aceito em: 08 Abr 2017. 


\section{INTRODUÇÃO}

Transtornos mentais são sabidamente problemas de saúde pública. De acordo com a Organização Mundial da Saúde (OMS), cerca de 650 milhões de indivíduos no mundo sofrem de algum tipo de transtorno mental, acarretando em sofrimento tanto para essas pessoas como para suas famílias e para a sociedade. Quando somados, tais transtornos são responsáveis por $25 \%$ dos anos vividos com incapacidade. ${ }^{1}$ Segundo o Ministério da Saúde, 3\% da população geral sofre de transtornos mentais graves e persistentes. ${ }^{2}$

Durante muitos anos, o tratamento dos transtornos psiquiátricos no Brasil consistiu em um modelo centrado na internação hospitalar, de longos períodos, que os privava do convívio social. Nos últimos anos diminuiu-se progressivamente a quantidade de leitos hospitalares e o tempo de internação, orientado-se a assistência através de serviços na rede básica de saúde. Esse novo modelo baseia-se no cuidado intensivo, personalizado e promotor de vida. Atualmente, a assistência ao paciente com transtornos mentais centraliza-se no convívio familiar. Neste contexto, surge o papel do cuidador como um dos responsáveis pela atenção, acompanhamento e reabilitação efetiva desses pacientes. ${ }^{3-5}$

O cuidador pode ser formal ou informal. O informal é a pessoa sem remuneração pelo papel desempenhado, responsável por auxiliar indivíduos doentes ou dependentes nas atividades diárias. ${ }^{6}$ Esse auxílio envolve cuidar das necessidades básicas, administrar medicamentos, fornecer suporte financeiro e ainda lidar com crises comportamentais. ${ }^{3}$ Diante do acúmulo dessas funções, esta ocupação acaba por trazer sobrecarga na vida desse indivíduo, podendo influenciar, inclusive, sua saúde. As dificuldades enfrentadas pelo cuidador são agravadas com o despreparo em lidar com pessoas com transtornos mentais, a falta de informações a respeito da doença, a falta de recursos no ambiente doméstico, as restrições da vida pessoal e o preconceito da sociedade. Isto acaba por gerar consequências negativas físicas e mentais, como hipertensão arterial, aumento dos níveis séricos de hormônios relacionados ao estresse, redução da imunidade e desenvolvimento de transtornos de humor. ${ }^{7,8}$

Reconhecendo a importância dessa população nos cuidados em saúde mental, é fundamental conhecer mais a respeito das características associadas à função de cuidador de pacientes com transtornos mentais. Assim, com este trabalho, objetivou-se conhecer o perfil dos cuidadores como uma forma de contribuir para a elaboração de ações voltadas a este grupo.

\section{MATERIAL E MÉTODOS}

Trata-se de um estudo transversal com cuidadores informais de pacientes dos ambulatórios de Psiquiatria Geral do Hospital Universitário Walter Cantídio da Universidade Federal do Ceará. Os cuidadores foram convidados a participar de uma entrevista no período de novembro a dezembro de 2016, quando responderam a um questionário sobre faixa etária, gênero, nível de escolaridade, estado civil, ocupação, grau de parentesco com o paciente, doenças, uso de medicamentos, tempo que se encaixa na função e nível de informações sobre o transtorno mental do paciente. A respeito dos pacientes, foram perguntados sobre o diagnóstico e os medicamentos em uso.

Os critérios de inclusão consistiram em os participantes terem mais de 18 anos e preencherem a definição de cuidador informal, sendo este o indivíduo responsável por auxiliar uma pessoa com doença mental ou dependente na realização de atividades diárias, sem receber remuneração por essa atividade.

Os dados coletados foram tabelados, analisados e apresentados sob formas de gráficos e tabelas, feitos com o auxílio do Microsoft Excel $^{\circledR}$ for Windows 2010, com o qual foram calculadas as médias das afirmações e os desvios.

Foram respeitados todos os princípios e normas para os procedimentos éticos em pesquisas envolvendo seres humanos requeridos pelo Comitê de Ética em pesquisa do Hospital Universitário Walter Cantídio (HUWC). Número do parecer do comitê de ética: 1.799.229.

\section{RESULTADOS}

68 cuidadores aceitaram serem entrevistados. A maioria pertencia ao sexo feminino $(80,9 \%)$, com idade variando entre 18 a 77 anos (média, 45,0; desvio padrão 15), tinha alguma relação de parentesco com o paciente e moravam no mesmo domicílio que o paciente. Dentre as relações de parentesco apontadas, destacaram-se filhos $(29,4 \%)$, pais (25\%), cônjuges $(22,1 \%)$ e irmãos (11,8\%). Sobre ocupação profissional, $54,4 \%$ disseram não trabalhar na época da entrevista e, destes, $57 \%$ disseram ter abandonado o trabalho para poder cuidar do paciente (Tabela 1$)$.

No tocante aos fatores relacionados a sua saúde, 26 (38,2\%) entrevistados disseram possuir doenças e, destes, apenas 6 $(23,1 \%)$ referiram possuir um transtorno mental. Do total de entrevistados, $42,6 \%$ relatou utilizar pelo menos um tipo de medicamento diariamente. Parte dos medicamentos de uso diário eram anticoncepcionais $(7,3 \%)$ ou suplementos vitamínicos $(4,4 \%)$.

A maioria exercia a função de cuidador há mais de 1 ano e cuidava de pacientes predominantemente do sexo feminino $(57,4 \%)$. Sobre informações recebidas em atendimentos, 26 $(38,2 \%)$ deles dizem não ter recebido explicações sobre o transtorno mental apresentado pelo paciente, 36 (52,9\%) não sabem como a doença pode evoluir ao longo do tempo e 44 $(64,7 \%)$ não receberam instruções sobre como auxiliá-lo em casa. Os principais diagnósticos psiquiátricos nos pacientes acompanhados foram depressão, transtornos psicóticos e transtorno bipolar (Tabela 2). As principais responsabilidades citadas foram: acompanhamento em consultas, administração de medicamentos, transporte, alimentação, exercícios, lazer e higiene, sendo que $95 \%$ deles são responsáveis por duas ou mais destas funções. A maioria dos pacientes utilizava diariamente mais que dois medicamentos $(82,3 \%)$. 
Tabela 1. Características sociodemográficas dos cuidadores de pacientes com transtornos mentais.

\begin{tabular}{|c|c|c|c|}
\hline Variáveis sociodemográficas & & $\mathrm{N}$ & $\%$ \\
\hline \multirow[t]{3}{*}{ Faixa Etária } & $18-40$ anos & 27 & 39,7 \\
\hline & $40-65$ anos & 34 & 50 \\
\hline & $>65$ anos & 7 & 10,3 \\
\hline \multirow[t]{2}{*}{ Gênero } & Masculino & 13 & 19,1 \\
\hline & Feminino & 55 & 80,9 \\
\hline \multirow[t]{7}{*}{ Escolaridade } & Analfabeto & 4 & 5,9 \\
\hline & Ensino fundamental incompleto & 17 & 25 \\
\hline & Ensino fundamental completo & 7 & 10,3 \\
\hline & Ensino médio incompleto & 3 & 4,4 \\
\hline & Ensino médio completo & 23 & 33,8 \\
\hline & Ensino superior incompleto & 5 & 7,4 \\
\hline & Ensino superior completo & 9 & 13,2 \\
\hline \multirow[t]{4}{*}{ Estado Civil } & Solteiro & 31 & 45,6 \\
\hline & Casado & 29 & 42,6 \\
\hline & Viúvo & 3 & 4,4 \\
\hline & Divorciado & 5 & 7,4 \\
\hline \multirow[t]{2}{*}{ Ocupação } & Sim & 31 & 45,6 \\
\hline & Não & 37 & 54,4 \\
\hline \multirow{3}{*}{$\begin{array}{l}\text { Depende financeiramente do } \\
\text { paciente }\end{array}$} & Sim, inteiramente dependente & 12 & 17,6 \\
\hline & Sim, parcialmente dependente & 13 & 19,1 \\
\hline & Não & 43 & 63,2 \\
\hline \multirow{2}{*}{$\begin{array}{l}\text { Possui relação de parentesco com o } \\
\text { paciente }\end{array}$} & Sim & 65 & 95,6 \\
\hline & Não & 3 & 4,4 \\
\hline
\end{tabular}

Tabela 2. Dados associados ao paciente e à função de cuidador.

\begin{tabular}{llll}
\hline Dados & & N & $\%$ \\
\hline Tempo que se encaixa na função de cuidador & Menos de 1 ano & 15 & 22,1 \\
& $1-5$ anos & 25 & 36,8 \\
& $6-10$ anos & 12 & 17,6 \\
& $11-15$ anos & 10 & 14,7 \\
& $16-20$ anos & 4 & 5,9 \\
& Mais que 20 anos & 2 & 2,9 \\
Sexo do paciente & Masculino & 29 & 42,6 \\
& Feminino & 39 & 57,4 \\
Principais diagnósticos psiquiátricos dos & Depressão & 23 & 33,8 \\
& Esquizofrenia & 21 & 30,9 \\
& Transtorno afetivo bipolar & 7 & 10,3 \\
& Transtorno de Ansiedade Generalizada & 7 & 10,3
\end{tabular}

Continua. 
Conclusão

Tabela 2. Dados associados ao paciente e à função de cuidador.

\begin{tabular}{llll}
\hline Dados & & $\mathrm{N}$ & $\%$ \\
\hline Algum profissional já lhe explicou sobre o que & Sim & 42 & 61,8 \\
é a doença do paciente & Não & 26 & 38,2 \\
Foi informado como a doença pode evoluir & Sim & 32 & 47,1 \\
& Não & 36 & 52,9 \\
Recebeu instruções sobre como ajudar o & Sim & 24 & 35,3 \\
paciente em casa & Não & 44 & 64,7 \\
Exerce a função de cuidador para outra pessoa & Sim & 11 & 16,2 \\
& Não & 57 & 83,8 \\
\hline
\end{tabular}

\section{DISCUSSÃO}

A maioria dos pacientes pertence ao sexo feminino, dado consistente com a literatura, que mostra ser esse o sexo predominante entre os pacientes com transtornos mentais. ${ }^{9}$ Em congruência com outros estudos acerca do perfil de cuidadores, ${ }^{10-14}$ notou-se o predomínio do gênero feminino além de algum grau de parentesco com o paciente. A função de cuidador, ao relacionar-se com a necessidade de suporte constante ao paciente, geralmente é atribuída a pessoas próximas, como pode ser observado pelo fato de residirem ambos no mesmo domicílio e por terem um parentesco próximo (mais de 75\% são filhos, pais ou cônjuges).

Ser cuidador envolve lidar com diversas decisões e dificuldades que surgem na rotina, o que pode ser facilitado se o indivíduo tiver informações adequadas a respeito dos transtornos. ${ }^{4}$ Entretanto, de acordo com os dados desde estudo, uma parcela importante $(38,2 \%)$ diz não ter recebido explicações sobre o transtorno mental apresentado pelo paciente. Este achado indica a falta de suporte oferecido ao cuidador, ainda mais quando levamos em conta que a grande maioria dos transtornos apresentados são comuns na rotina dos atendimentos em saúde em qualquer nível de assistência. Espera-se uma psicoeducação adequada à população, e por isso este dado chama a atenção, pois a maioria dos cuidadores não sabe do que se trata o transtorno do paciente.

Entender como a doença se manifesta ao longo do tempo ajuda no processo do cuidado ao paciente com transtorno mental.

\section{REFERÊNCIAS}

1. World Health Organization. Investing in mental health: evidence for action [Internet]. Geneva: WHO; 2013 [Acesso em: 27 jul 2016]. Disponível em: http://apps.who.int/iris/ bitstream/10665/87232/1/9789241564618_eng.pdf

2. Brasil. Ministério da Saúde. Diretrizes assistenciais em saúde mental na saúde suplementar [Internet]. Brasília: Ministério da Saúde; 2008 [Acesso em: 18 jul 2016]. Disponível em: http://www. ans.gov.br/images/stories/Plano_de_saude_e_Operadoras/Area_do_ consumidor/diretrizes_assistenciais.pdf

3. Tabeleão VP; Tomasi E; Quevedo LA. Sobrecarga de familiares
Diante da falta de explicações básicas sobre o diagnóstico, era esperado uma carência geral nas demais informações, sendo isto confirmado pelas porcentagens ainda maiores de cuidadores que não foram informados sobre como a doença poderia evoluir e sobre como auxiliar o paciente em casa. Expectativas não realistas de independência do paciente para atividades gerais e as frustrações advindas disto podem ser a causa de sobrecarga e sofrimento do cuidador. Tendo em vista que muitas das condições psiquiátricas apresentadas podem gerar incapacidades, oferecer explicações e explorar possíveis dúvidas durante as consultas permitiria diminuir possíveis respostas negativas às dificuldades rotineiras do paciente. Ademais, a literatura comprova que intervenções em grupo para cuidadores, seja no aspecto psicoeducacional ou psicoterapêutico, são importantes para diminuir os níveis de estresse e sobrecarga. ${ }^{15}$

Além disso, o cuidado do paciente não pode se restringir ao momento da consulta. Em conjunto com o planejamento de ações mais imediatas (medicamentos, exames etc) é necessário oferecer uma visão integral do paciente, permitindo que o cuidador em sua rotina diversifique seu modo de cuidar.

Diante do exposto, evidencia-se a importância de que os profissionais destinem maior atenção ao cuidador para que este compreenda o transtorno mental e exerça a sua função da melhor forma, evitando consequências negativas e trazendo benefícios para os envolvidos. Como limitação deste estudo destaca-se o fato dele ter sido realizado somente no Hospital Universitário Walter Cantídio e representar o perfil dos cuidadores apenas desta instituição.

de pessoas com transtorno psíquico: níveis e fatores associados. Rev Psiquiatr Clín. 2014;41(3):63-6.

4. Nolasco M, Bandeira M, Oliveira MS, Vidal, CE. Sobrecarga de familiares cuidadores em relação ao diagnóstico de pacientes psiquiátricos. J Bras Psiquiatr. 2014;63(2):89-97.

5. Albuquerque EP, Cintra AM, Bandeira M. Sobrecarga de familiares de pacientes psiquiátricos: comparação entre diferentes tipos de cuidadores. J Bras Psiquiatr. 2010;59(4):308-16.

6. Brasil. Ministério da Saúde. Guia prático do cuidador [Internet]. 
Brasília: Ministério da Saúde; 2008 [Acesso em: 16 jul 2016]. Disponível em: http://bvsms.saude.gov.br/bvs/publicacoes/guia_ pratico_cuidador.pdf

7. Cardoso L, Galera SA, Vieira MV. O cuidador e a sobrecarga do cuidado à saúde de pacientes egressos de internação psiquiátrica. Acta Paul Enferm. 2012;25(4):517-23.

8. Valente L, Truzzi A, Souza WF, Alves GS, Sudo FK, Alves CE, et al. Autopercepção de saúde em cuidadores familiares e o tipo de demência: resultados preliminares de uma amostra ambulatorial. Rev Bras Neurol. 2013;49(1):13-9.

9. Santos EG, Siqueira MM. Prevalência dos transtornos mentais na população adulta brasileira: uma revisão sistemática de 1997 a 2009. J Bras Psiquiatr. 2010;59(3):238-46.

10. Oliveira DC, Carvalho GS, Stella F, Higas CM, Elboux MJ. Qualidade de vida e sobrecarga de trabalho em cuidadores de idosos em seguimento ambulatorial. Texto Contexto Enferm. 2011;20(2):234-40.
11. Almeida MM, Schal VT, Martins AM, Modena CM. A sobrecarga de cuidadores de pacientes com esquizofrenia. Rev Psiquiatr Rio Gd Sul. 2010;32(3):73-9.

12.Barroso SM, Bandeira M, Nascimento E. Sobrecarga de familiares de pacientes psiquiátricos atendidos na rede pública. Rev Psiquiatr Clín. 2007;34(6):270-7.

13. Gaioli CC, Furegato AR, Santos JL. Perfil de cuidadores de idosos com doença de Alzheimer associado à resiliência. Texto Contexto Enferm. 2012;21(1):150-7.

14. Moscoso MA, Marques RC, Ribeiz SR, Santos L, Bezerra DM, Jacob W Filho, et al. Profile of caregivers of Alzheimer's disease patients attended at a reference center for cognitive disorders. Dement Neuropsychol. 2007;1(4):412-17.

15. Santos RL, Sousa MF, Brasil D, Dourado M. Intervenções de grupo para sobrecarga de cuidadores de pacientes com demência: uma revisão sistemática. Rev Psiquiatr Clín. 2011;38(4):161-7.

\section{Como citar:}

Santos JP, Araújo LC, Timbó SR, Costa RF, Girão JE, Lima AA, et al. Perfil dos cuidadores de pacientes com transtornos mentais do Hospital Universitário Walter Cantídio. Rev Med UFC. 2018 abr-jun;58(2):14-18. 